\title{
Validité et fidélité de la version française d'un questionnaire portant sur le choix de carrière des étudiants en médecine
}

\author{
Validity and reliability of the French version of a questionnaire on career \\ choices of medical students and their inclination towards primary care
}

\author{
Marie-Dominique Beaulieu ${ }^{1}$, Jeannie HaGgerty ${ }^{2}$, Fatima Bouharaoui ${ }^{3}$ \\ et François GoulET ${ }^{4}$
}

1 Chaire Docteur Sadok Besrour en médecine familiale, Centre de recherche du Centre hospitalier de l'Université de Montréal (CHUM) ; Département de médecine familiale et de médecine d'urgence, Faculté de médecine, Université de Montréal, Montréal, Canada

2 Chaire de recherche du Canada sur l'impact des services de santé sur la population, Université Sherbrooke ; Centre de recherche de l'Hôpital Charles LeMoyne, Longueuil, Canada

3 Centre de recherche de l'Hôpital Charles LeMoyne, Longueuil, Canada

4 Département de médecine familiale et de médecine d'urgence, Faculté de médecine, Université de Montréal ; Collège des médecins du Québec, Canada

Manuscrit reçu le 20 mai 2009 ; commentaires éditoriaux formulés aux auteurs le 6 février 2010 ; accepté pour publication le 10 mai 2010

\begin{abstract}
Mots clés :
Étudiants en médecine ;

choix de carrière ;

médecine familiale ;

médecine générale ;

questionnaire ;

validation

Résumé - Contexte : La diminution constante, dans tous les pays industrialisés, du nombre d'étudiants en médecine qui optent pour une carrière en médecine générale suscite un intérêt marqué pour la compréhension des facteurs qui influent sur ces choix. Peu d'études s'appuient sur des instruments validés. La disponibilité de tels instruments en français est inexistante. But : Évaluer la fidélité d'une traduction française du questionnaire validé de Wright et al., conçu pour mesurer les facteurs associés aux choix de carrière des étudiants en médecine. Sujets/matériel/méthodes : Après une étape de traduction/retraduction, cent soixante-neuf étudiants terminant leur premier cycle d'études dans les quatre facultés de médecine de la province de Québec ont complété une version Web du questionnaire français. Des analyses factorielles de même que des analyses de régression logistique ont été employées pour évaluer la validité conceptuelle et prédictive de la version française produite. Résultats : La résolution factorielle obtenue lors de l'analyse exploratoire différait légèrement de celle des auteurs de la version originale du questionnaire quant au nombre de facteurs et leur composition. Les différences observées peuvent être imputées à la surreprésentation d'étudiants ayant opté pour la médecine familiale dans notre échantillon. La résolution factorielle exploratoire et celle obtenue en contraignant la résolution factorielle de la version originale expliquent une proportion identique de la variance du choix de carrière de nos participants, soit $50 \%$. Conclusion : Notre traduction française du questionnaire de Wright est aussi valide et fiable que la version originale et peut être utilisée dans le cadre d'études visant des étudiants francophones.
\end{abstract}




$\begin{array}{ll}\text { Keywords: } & \text { Abstract - Context: The steady decline in the number of medical students choosing a ca- } \\ \text { Medical students; } & \text { reer in general medicine has been observed in all industrialized countries. There is significant } \\ \text { career choice; } & \text { interest in understanding the factors that influence these choices. Few studies are based on } \\ \text { family medicine; } & \text { validated instruments and no such instrument is available in French. Objective: To evalu- } \\ \text { general medicine; } & \text { ate the reliability of the French version of a validated questionnaire designed to assess the } \\ \text { questionnaire } & \text { factors associated with medical students' career choices. Participants/material/methods: } \\ \text { validation } & \text { Following the translation and retranslation of Wright et al.'s questionnaire, one hundred and } \\ & \text { sixty-nine medical students completing their undergraduate medical program in the four Que- } \\ \text { bec faculties of medicine completed a Web-based French version of the questionnaire. Factor } \\ \text { analyses and logistic regressions were used to assess construct and predictive validity of the } \\ \text { French version. Results: The exploratory factor analysis produced a factor solution slightly } \\ \text { different from that of the authors of the original questionnaire in terms of number of factors } \\ \text { and factor structure. These differences can be attributed to the overrepresentation of students } \\ \text { choosing family medicine in the sample. The exploratory factorial solution and that obtained } \\ \text { by forcing the original authors' factorial solution predicted an identical proportion of the vari- } \\ \text { ance associated with participants' career choices (50\%). Conclusions: Our French translation } \\ \text { of Wright's questionnaire is as valid and reliable as the original and may be used in studies } \\ \text { aimed at French-speaking respondents. }\end{array}$

\section{Introduction}

Dans tous les pays industrialisés, on observe une diminution constante du nombre d'étudiants en médecine qui optent pour une carrière en «médecine générale » ${ }^{[1-4]}$, ce terme étant entendu, dans le cadre de cette étude, comme synonyme de «médecine familiale », en contraste avec le «primary care » aux États-Unis qui inclut certaines spécialités telles que la pédiatrie, l'obstétrique/gynécologie et la médecine interne. Une récente étude réalisée en France auprès de 600 étudiants en préparation pour les épreuves classantes nationales rapporte ainsi que seulement $11,3 \%$ d'entre eux ont fait de la médecine générale leur choix de carrière ${ }^{[5]}$. La compréhension des caractéristiques qui influencent ce choix suscite un grand intérêt et la recherche a produit sur le sujet des résultats parfois non concluants, voire contradictoires. Les chercheurs s'entendent toutefois généralement pour classer ces facteurs selon trois grandes catégories : les caractéristiques sociodémographiques des étudiants (âge; sexe; région d'origine); les caractéristiques des facultés et des programmes de médecine, enfin l'interaction entre les caractéristiques des spécialités et les attitudes et valeurs personnelles des étudiants ${ }^{[6-10]}$.

Mesurer de façon fiable et valide des attitudes et des valeurs représente un défi méthodologique important. Parmi la myriade d'études effectuées sur les choix de carrière des étudiants en médecine au cours des dernières années, très peu s'appuient sur des instruments robustes. Au début des années 90, Beaudoin et al. ${ }^{[11]}$ avaient élaboré et validé un questionnaire (trois échelles, 13 items) qui ne visait pas tant à prédire les choix de carrière qu'à évaluer les différences dans les orientations des médecins à l'égard de divers aspects des soins, intégrés selon les caractéristiques de leur formation (résidence ou internat). En 2004, Ward et al. ${ }^{[12]}$ ont utilisé le questionnaire Attitudes to Social Issues in Medicine (ATSIM) ${ }^{[13]}$ pour déceler les facteurs précoces prédictifs des choix de carrière. Ce questionnaire, qui comporte 63 items, ne mesure cependant que les attitudes à l'égard des dimensions sociales des soins comme prédicteurs des choix de carrière.

Deux auteurs ont élaboré et validé des questionnaires portant sur un ensemble plus complet 
d'attitudes et de valeurs des étudiants en médecine relativement à leur carrière médicale future. Aux États-Unis, Murdoch et al. ont validé un questionnaire en anglais de 46 items comportant sept échelles : orientation bioscientifique; orientation biosociale; intérêt académique; prestige; revenu; évitement des contraintes et rôle de soutien ${ }^{[14]}$. Au Canada, Wright et al. ${ }^{[15]}$ ont conçu un questionnaire anglais de 25 items à partir d'une analyse bibliographique et d'entretiens qualitatifs avec des étudiants et des enseignants. Les items sont regroupés sous cinq facteurs : style de vie médicale (medical lifestyle), orientation sociétale (societal orientation), prestige, orientation hospitalière (hospital orientation) et diversification du champ de pratique (varied scope of practice). Dans le cadre de leurs recherches sur la mise au point d'un modèle expliquant la variance du choix de carrière des étudiants en médecine (médecine générale vs. médecine spécialisée), le questionnaire de Murdoch a prédit $43 \%$ de la variance $^{[14]}$ tandis que le questionnaire de Wright en a prédit $52 \%{ }^{[15]}$. Murdoch et al. avaient questionné l'ensemble des étudiants de la University of Minnesota School of Medicine sans égard à leur stade d'avancement dans le programme $(n=670)$, alors que Wright et al. avaient interrogé des étudiants en médecine de trois universités canadiennes au moment de leur entrée dans le programme de médecine (formation premier cycle) $(n=519)$. Finalement, deux autres questionnaires largement utilisés au Canada abordent également cette problématique : le sondage national auprès des médecins du Collège des médecins de famille du Canada ${ }^{[16]}$ et le Canadian Resident Matching Service (CaRMS) ${ }^{[17]}$. Ces questionnaires ont des versions différentes selon le statut du répondant auquel ils s'adressent (étudiants aux études de premier cycle ou de deuxième cycle, professionnel en pratique). Bien qu'ils reprennent certaines des questions de Wright et al., ces questionnaires n'ont toutefois pas été validés dans leurs versions intégrales anglaise et française.

L'analyse de la littérature ne relève donc aucun questionnaire validé en français sur le choix de carrière des étudiants en médecine. Ainsi, les auteurs d'une étude récente ${ }^{[18]}$ sur les choix de spécialité d'étudiants en médecine avaient dû laisser la portion de leur questionnaire portant sur les valeurs associées aux choix de carrière (tirée du questionnaire de Murdoch) en anglais, alors que les autres parties étaient en français, faute d'une version francophone validée disponible. Pourtant, les problématiques liées aux choix de carrière en médecine touchent tout autant les pays francophones qu'anglophones. En effet, le déclin en popularité de la médecine générale auprès des futurs médecins s'observe non seulement en France ${ }^{[5,19,20]}$ et en Belgique ${ }^{[21]}$, mais demeure une préoccupation également dans des pays comme la Tunisie ${ }^{[22]}$ et le Liban ${ }^{[18]}$, où l'organisation des soins de première ligne est en voie de développement. Dans ce contexte, un questionnaire français validé s'avérerait être un outil précieux.

En 2005, nous avons effectué une traduction française du questionnaire de Wright afin de mener une enquête sur le choix de carrière (médecine générale vs. spécialisée) de tous les étudiants en médecine dans les quatre facultés de médecine de la province de Québec. Cet article présente les résultats des analyses de fidélité et de validité effectuées sur la version française du questionnaire.

\section{Matériel et méthodes}

\section{Population étudiée}

Le premier cycle des études médicales au Québec est d'une durée de quatre à cinq ans selon que l'étudiant ait eu à faire l'année préparatoire de médecine ou non. À la fin du second trimestre de la dernière année de ce premier cycle, les étudiants choisissent le programme de résidence (formation de deuxième cycle) qui correspond à leur choix de carrière. En mai 2005, une enquête a été menée auprès de tous les étudiants terminant leur premier cycle d'études médicales au Québec en juin $2005(n=565)$, c'està-dire une fois leur choix fait et confirmé. Les directeurs des stages cliniques des différentes universités ont envoyé à ces finissants une invitation à participer 
à l'enquête ainsi qu'une lettre expliquant les objectifs de l'étude. Les étudiants étaient dirigés vers un site Web interactif sur lequel ils pouvaient répondre à la version française du questionnaire. Deux rappels ont été envoyés à deux semaines d'intervalle. Cent soixante-neuf étudiants ont complété le questionnaire (taux de réponse de $30 \%$ ). Le projet a reçu l'approbation du comité d'éthique de la recherche de la faculté de médecine de l'Université de Montréal.

\section{Questionnaire}

Le questionnaire de Wright ${ }^{[15]}$ a été privilégié parmi les quelques autres questionnaires disponibles pour différentes raisons : il avait déjà fait l'objet d'une validation en anglais; il était parmi les plus courts ; il englobait cinq dimensions distinctes dont on sait qu'elles influencent le choix de carrière des étudiants en médecine; il avait démontré une bonne validité prédictive. Le questionnaire est subdivisé en trois parties : 1) choix de programme et intentions quant à la résidence ; 2 ) facteurs influençant le choix de carrière ; 3) renseignements personnels. Les réponses aux items sont cotées selon une échelle de Likert à cinq points allant de 1 (aucune influence) à 5 (grande influence). La procédure de traduction s'est déroulée en cinq étapes inspirées de la méthode de Beaton et al. ${ }^{[23]}$, qui proposent une marche à suivre pour adapter des questionnaires auto-administrés dans différentes langues et cultures. En raison de la relative proximité culturelle des personnes visées par les versions respectivement originale (culture canadienne-anglaise) et traduite (culture canadienne-française), l'équipe de chercheurs, maîtrisant les deux langues et les concepts et mesures, a agi à titre de point de comparaison informé pour proposer des ajustements suite aux étapes de traduction et retraduction, plutôt que d'avoir recours à deux traducteurs différents dans chaque langue. Ainsi, la version originale a d'abord été traduite en français par un traducteur francophone professionnel (étape 1 : traduction). Les chercheurs ont discuté de cette première traduction et proposé plusieurs ajustements, pour donner lieu à une version «améliorée » (étape 2 : synthèse). Cette version traduite « améliorée » a ensuite été retraduite vers l'anglais par un traducteur anglophone professionnel (étape 3 : retraduction). La retraduction anglaise a été comparée à la version originale par le groupe de chercheurs pour vérifier s'il y avait des différences de signification (étape 3 : révision par un comité expert). Aucune différence marquante n'a été relevée. La version française «améliorée » a finalement été pré-testée sur quinze étudiants québécois francophones afin de finaliser l'adaptation du questionnaire original (étape 5 : pré-test). Nous n'avons conservé, dans la version française, que les 17 items (sur 25 au total) qui faisaient partie de la résolution factorielle de l'étude de Wright et al., huit items du questionnaire ne s'étant avérés corrélés à aucun facteur dans leur étude ${ }^{[15]}$. Trois items ont été ajoutés aux 17 retenus : ils portent sur l'attrait de la pratique en milieu urbain plutôt qu'en milieu rural et sur la durée de la résidence. Ces enjeux étaient particulièrement importants au Québec au moment de l'étude, où il était notamment question de prolonger d'un an la résidence en médecine familiale. Les items non validés du Sondage national et du CaRMS s'adressant aux étudiants à la fin du premier cycle d'études médicales et ne figurant pas dans le questionnaire de Wright ont été examinés. Ces items ne correspondaient à aucun nouveau concept et n'offraient aucune valeur ajoutée en lien avec l'investigation du choix de carrière des étudiants en médecine, ne justifiant donc pas l'allongement d'un questionnaire dont une des qualités visées était justement sa concision.

\section{Analyses}

La validité conceptuelle de la version française a d'abord fait l'objet d'une analyse factorielle exploratoire $^{[24]}$. Le nombre de facteurs retenus a été déterminé en fonction du critère de Kaiser (valeurs propres des facteurs $>1$ ). La factorisation par la méthode d'analyse factorielle des correspondances, qui recherche les principaux facteurs permettant d'expliquer la variance commune aux items, a été employée avec rotation oblique des facteurs. Ce type de rotation permet qu'il y ait corrélation entre les 
facteurs et s'avérait donc plus approprié au phénomène étudié. Nous avons ensuite effectué une analyse factorielle contraignant les items selon les cinq échelles originales de Wright afin d'en vérifier la stabilité sur notre population avec le questionnaire traduit. Les facteurs ont fait l'objet d'analyses descriptives, incluant l'analyse des inter-corrélations et le calcul des coefficients alpha $(\alpha)$ de Cronbach. Nous avons ensuite comparé la validité prédictive quant au choix de carrière déclaré par les étudiants des deux solutions factorielles obtenues (exploratoire et «contrainte »). Des régressions logistiques, prenant pour variable dépendante «avoir sélectionné la médecine familiale comme premier choix de résidence par opposition à avoir sélectionné une autre spécialité comme premier choix », ont été employées à cette fin, les étudiants canadiens et québécois sélectionnant parfois plus d'un programme pour s'assurer d'une place en résidence. Le degré de corrélation entre les variables indépendantes du modèle a été examiné au préalable, afin d'écarter tout risque de colinéarité. Des tests de corrélation de Spearman ont été effectués à cette fin, car les associations n'étaient pas linéaires ${ }^{[25]}$. Les analyses ont été effectuées sur le logiciel SAS v9.1 ${ }^{\circledR}$.

\section{Résultats}

L'analyse porte sur les 169 étudiants ayant complété le questionnaire en ligne. Le tableau I présente certaines de leurs caractéristiques sociodémographiques.

Analyse factorielle exploratoire et comparaison des solutions factorielles

L'analyse factorielle exploratoire a produit quatre facteurs plutôt que cinq comme l'avaient spécifié Wright et al. dans leur article original. La composition des facteurs que nous avons obtenus diffère également légèrement de la leur (tableau II). Deux items associés au facteur «style de vie médicale » dans le questionnaire anglais ne se sont pas avérés associés à ce facteur dans notre analyse : «flexibilité médicale (choisir ce que je veux faire en médecine) » n'a été associé à aucun facteur et l'item «me laisser des ouvertures pour l'avenir » a plutôt été associé au facteur «orientation sociétale », même s'il s'agissait de l'item contribuant le moins à ce facteur (coefficient factoriel $=0,49$ ). L'analyse exploratoire a également saturé sur un même facteur les questions portant sur l'intérêt des étudiants vis-à-vis de la pratique hospitalière et celles explorant le prestige perçu de la carrière choisie, alors qu'elles constituaient deux facteurs distincts dans l'étude de Wright. De plus, la question portant sur l' «accent mis sur les soins d'urgence » s'est avérée associée à deux facteurs : au facteur combiné «prestige et orientation hospitalière » (coefficient factoriel $=0,51$ ) et à celui de «diversification du champ de pratique » (coefficient factoriel $=0,53$ ). Ceci dénote un item potentiellement complexe et nous avons choisi de l'associer au facteur «diversification du champ de pratique » dans notre modèle, alors qu'il était associé au facteur d'attirance pour la médecine hospitalière dans l'étude de Wright. Ce quatrième facteur («diversification du champ de pratique ») s'est composé de six items, comparativement à seulement deux items pour Wright, les items additionnels étant l'«accent mis sur les soins d'urgence » (tel qu'indiqué précédemment), ainsi que les trois ajoutés dans la version française du questionnaire. Deux items associés négativement au facteur ont été recodés inversement : « se concentrer sur une moins grande diversité de problèmes posés par les patients et/ou sur un groupe d'âge précis » et «maximiser les chances de pratiquer dans un grand centre urbain ».

Comparaison de la validité prédictive des deux solutions

Les variables suivantes ont été incluses d'un seul bloc dans les analyses de régression logistique : sexe; âge; statut civil; montant des dettes et les quatre ou cinq facteurs selon le modèle analysé. Le choix de l'université n'était aucunement associé au choix de la médecine familiale. Les résultats sont rapportés sous forme de rapports de cotes (RC) avec un intervalle de confiance (IC) de $95 \%$. Le rapport de cotes («odds ratio » en anglais) se calcule comme 
Tableau I. Caractéristiques sociodémographiques des étudiants en médicine ayant répondu au questionnaire, selon leur choix de la médecine familiale ou d'une autre spécialité.

\begin{tabular}{|c|c|c|c|}
\hline & $\begin{array}{l}\text { Choix de la médecine } \\
\text { familiale } \\
(n=119)\end{array}$ & $\begin{array}{l}\text { Choix d'une autre } \\
\text { spécialité médicale } \\
(n=50)\end{array}$ & Valeurs $p$ \\
\hline$\%$ Sexe féminin & $98(82,3 \%)$ & $31(63,3 \%)$ & 0,008 \\
\hline Moyenne d'âge (écart-type) & 24,8 années $(3,1)$ & 24,9 années $(3,1)$ & 0,59 \\
\hline $\begin{array}{l}\text { Statut civil : } \\
\text { Célibataire } \\
\text { En couple }\end{array}$ & $\begin{array}{l}81(68,6 \%) \\
37(31,4 \%)\end{array}$ & $\begin{array}{l}34(69,4 \%) \\
15(30,6 \%)\end{array}$ & 0,92 \\
\hline $\begin{array}{l}\text { Population dans la } \\
\text { communauté où les études } \\
\text { secondaires ont été } \\
\text { complétées : } \\
\quad<50000 \\
50000-99999 \\
100000-500000 \\
>500000\end{array}$ & $\begin{array}{l}44(37 \%) \\
15(13 \%) \\
15(13 \%) \\
44(37 \%)\end{array}$ & $\begin{array}{l}17(35 \%) \\
5(10,2 \%) \\
5(10,2 \%) \\
22(44,6 \%)\end{array}$ & 0,63 \\
\hline $\begin{array}{l}\text { Éducation avant les études } \\
\text { médicales : } \\
\text { Diplôme d'études } \\
\text { collégiales (formation } \\
\text { pré-universitaire) } \\
\text { Premier cvcle universitaire }\end{array}$ & $\begin{array}{l}76(63,8 \%) \\
34(28.6 \%)\end{array}$ & $\begin{array}{l}33(66 \%) \\
12(24 \%)\end{array}$ & 0,90 \\
\hline $\begin{array}{l}\text { Déquivalant baccalauréat) } \\
\text { cycle universitaire } \\
\text { (équivalant maîtrise/doctorat) }\end{array}$ & $\begin{array}{l}34(28,6 \%) \\
8(6,7 \%)\end{array}$ & $4(8 \%)$ & 0,90 \\
\hline $\begin{array}{l}\text { Université : } \\
\text { - Montréal } \\
\text { - McGill } \\
\text { - Sherbrooke } \\
\text { - Laval }\end{array}$ & $\begin{array}{l}16(13,6 \%) \\
32(27,3 \%) \\
46(39,3 \%) \\
23(19,6 \%)\end{array}$ & $\begin{array}{l}8(16,3 \%) \\
15(30,6 \%) \\
18(37,7 \%) \\
8(16,3 \%)\end{array}$ & 0,90 \\
\hline $\begin{array}{l}\text { Dette étudiante } \\
\begin{aligned} \text { (en dollars canadiens) : } \\
\text { - }<\$ 10000 \\
\text { - } \$ 10000-\$ 29999 \\
\text { - } \$ 30000-\$ 49999 \\
\text { - } \geq \$ 50000\end{aligned}\end{array}$ & $\begin{array}{l}35(29,4 \%) \\
55(46,2 \%) \\
15(12,6 \%) \\
14(11,8 \%)\end{array}$ & $\begin{array}{l}17(34,7 \%) \\
23(46,9 \%) \\
3(6,1 \%) \\
6(12,2 \%)\end{array}$ & 0,64 \\
\hline $\begin{array}{c}\text { Dette non liée aux études : } \\
\square<\$ 10000 \\
\square \$ 10000-\$ 29999 \\
\square \$ 30000-\$ 49999 \\
\square \geq \$ 50000\end{array}$ & $\begin{array}{l}63(52,9 \%) \\
36(30,3 \%) \\
7(5,9 \%) \\
13(10,9 \%)\end{array}$ & $\begin{array}{l}30(61,2 \%) \\
10(20,4 \%) \\
5(10,2 \%) \\
4(8,2 \%)\end{array}$ & 0,42 \\
\hline
\end{tabular}


Tableau II. Comparaison des résultats de l'analyse factorielle exploratoire sur la version française du questionnaire avec la résolution factorielle des auteurs de la version originale ${ }^{[15]}$.

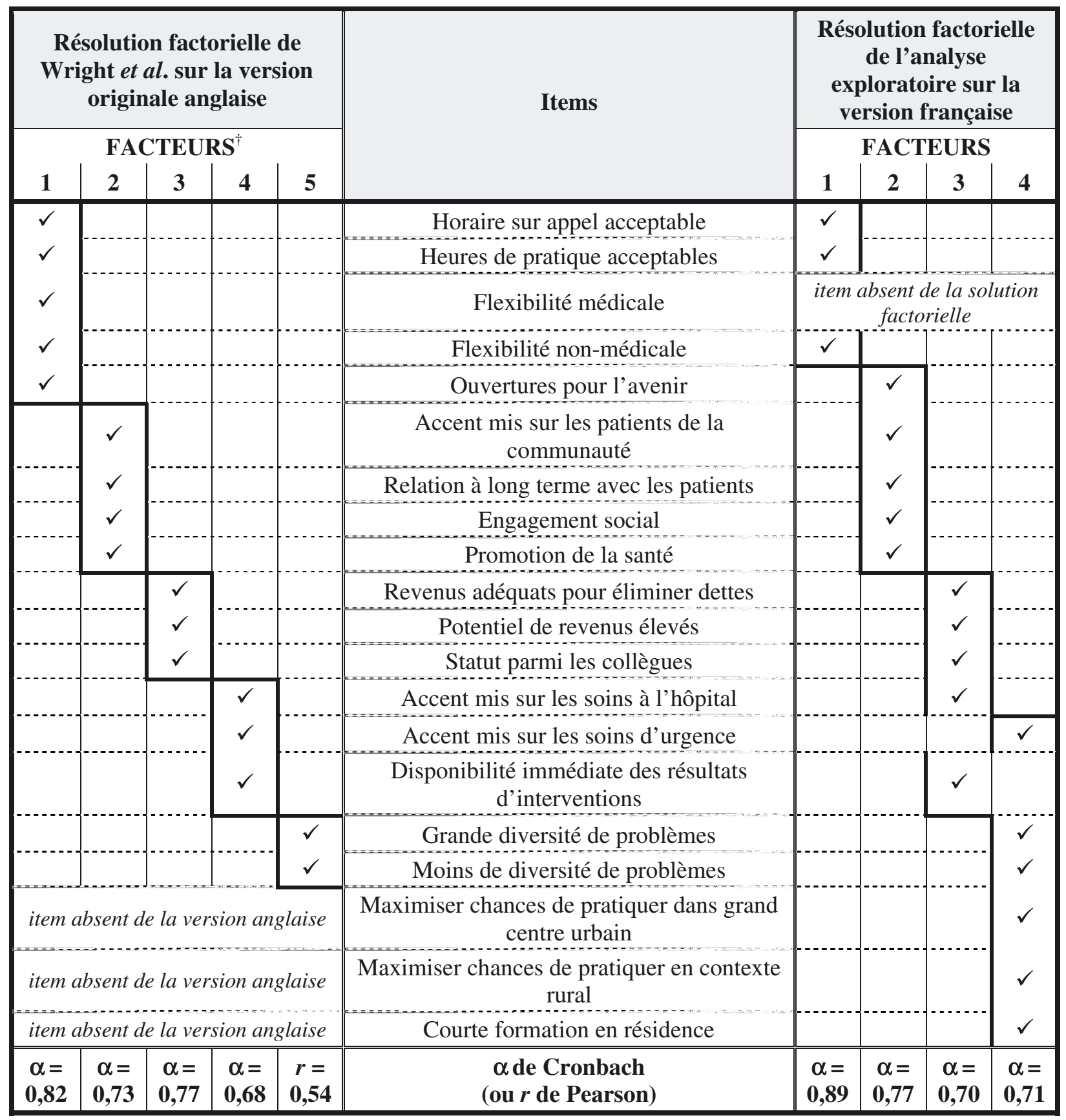

${ }^{\dagger}$ Noms donnés aux facteurs par Wright et al. : 1 = style de vie médicale; 2 = orientation sociétale; $3=$ prestige; $4=$ orientation hospitalière; $5=$ diversification du champ de pratique. 
le ratio entre la probabilité de présence d'une condition («avoir choisi la médecine familiale comme premier choix au terme du premier cycle d'études médicales ») et la probabilité de non-présence ( $p / 1$ p) selon la variable mesurée. Un rapport de cote se situant entre 0 et 1 signifie donc que la variable considérée est associée à une probabilité moindre d'avoir choisi la médecine familiale (c.-à-d. que les étudiants ayant cette caractéristique ont moins de chance d'avoir choisi la médecine familiale que d'avoir choisi une autre spécialité comme premier choix), alors qu'une valeur supérieure à 1 représente une probabilité supérieure d'avoir choisi la médecine familiale. Un rapport de cotes égal à 1 signifie que la variable considérée est associée à une probabilité égale d'avoir choisi la médecine familiale ou non : elle n'est donc associée d'aucune façon au choix de la médecine familiale et en est indépendante.

Dans notre modèle avec les variables sociodémographiques et quatre facteurs, l'analyse a montré que deux variables seulement étaient associées de façon significative au choix de la médecine familiale : les facteurs « orientation sociétale » $[\mathrm{RC}: 2,94$ $(1,61-5,37)]$ et «diversification du champ de pratique » $[\mathrm{RC}: 6,31(2,915-13,64)]$. Ce modèle expliquait $50,0 \%$ de la variance quant au choix de la médecine familiale ou non au terme du premier cycle d'études médicales. Dans le modèle avec cinq facteurs calqués sur ceux de Wright, trois variables étaient associées de façon significative au choix de la médecine familiale : les facteurs « orientation sociétale » $[\mathrm{RC}: 2,58(1,51-4,41)]$, « orientation hospitalière » $[R C: 1,80(1,10-2,95)]$, et « diversification du champ de pratique » $[\mathrm{RC}: 3,66(1,85-7,26)]$. Ce dernier modèle expliquait $49,8 \%$ de la variance. Notons qu'aucune des variables sociodémographiques ne s'est avérée significativement associée à la variable dépendante dans les analyses à quatre ou cinq facteurs.

\section{Discussion}

Nos résultats permettent d'évaluer la fidélité et la validité de la version française du questionnaire de
Wright et al. relatif aux valeurs des étudiants en médecine associées au choix d'une carrière en médecine générale. La résolution factorielle obtenue dans notre étude diffère légèrement de celle des auteurs de la version originale anglaise ${ }^{[15]}$. Nous avons obtenu quatre facteurs et non cinq, «prestige » et « orientation hospitalière » étant combinés sous un même facteur. Autre différence notable, nous avons intégré l' « accent sur les soins d'urgence » dans le facteur « diversification du champ de pratique » plutôt que dans le facteur combiné «prestige et orientation hospitalière », compte tenu de sa contribution légèrement plus forte à ce facteur. Conceptuellement, l'intérêt pour les soins d'urgence peut être lié aux deux facteurs : il s'agit certes d'une pratique hospitalière mais elle se situe à la jonction des soins de santé primaires et offre une grande variabilité des cas à gérer. Malgré ces différences, les modèles à quatre et cinq facteurs ont présenté une validité prédictive très similaire, le modèle à quatre facteurs expliquant $50,0 \%$ de la variance associée au choix de la médecine familiale dans notre échantillon, comparativement à $49,8 \%$ pour la solution à cinq facteurs, qui expliquait $52 \%$ de la variance dans l'étude de Wright ${ }^{[15]}$. Selon les deux modèles, $l^{\prime}$ « orientation sociétale » et la «diversification du champ de pratique » se sont avérés être les facteurs les plus fortement associés au choix d'une carrière en médecine familiale, tout comme cela était le cas dans l'étude de Wright ${ }^{[15]}$.

Nous ne croyons pas que l'administration du questionnaire par voie électronique ait introduit un biais particulier à notre étude. Il a été clairement démontré que, pour le type de population que nous voulions rejoindre, les caractéristiques des répondants ne variaient pas selon que le questionnaire soit administré sur le Web, par la poste ou par téléphone ${ }^{[26]}$. En revanche, le taux de réponse que nous avons obtenu $(30 \%)$ constituerait une limite très importante si nous avions voulu généraliser le choix de carrière de notre échantillon à l'ensemble de la population des étudiants en médecine du Québec. Même si notre objectif était d'évaluer la fidélité de notre version française du questionnaire de Wright, 
il nous faut considérer les principales différences observées entre nos résultats et ceux de Wright à l'aune des caractéristiques de nos échantillons respectifs.

Dans l'échantillon de Wright, $80 \%$ des répondants avaient opté pour une spécialité médicale comme premier choix de carrière ${ }^{[15]}$, comparativement à seulement $30 \%$ des répondants de notre échantillon. Les énoncés relatifs au prestige et à l'attirance pour la pratique hospitalière se seraient sans doute distribués différemment si nous avions obtenu davantage d'étudiants intéressés par les spécialités car nous savons que ces facteurs sont associés plus fréquemment à ce choix de carrière ${ }^{[6-10]}$. À ce sujet, il convient de mentionner que dans l'étude de Wright, le facteur «prestige » s'était avéré être le seul, parmi les cinq dégagés, à ne pas affecter significativement la préférence (les auteurs questionnaient les étudiants à leur entrée dans le programme de médecine) pour une carrière en médecine familiale. Lors des analyses de régression logistique avec notre modèle à quatre facteurs, le facteur combiné «prestige et orientation hospitalière » n'avait aucune influence significative sur la probabilité de choisir la médecine familiale, alors qu'avec le modèle à cinq facteurs, l'orientation hospitalière s'avérait être un prédicteur significatif. Nous pouvons donc penser que la combinaison des facteurs de «prestige » et d' «attirance pour la pratique hospitalière » ait pu diluer sous le seuil de signification l'impact de certains items d'orientation hospitalière. De la même manière, il peut être souhaitable de maintenir la distinction entre ces deux facteurs d'un point de vue conceptuel. En effet, l'attrait de la médecine hospitalière et la place qu'elle prend dans la pratique de la médecine familiale sont susceptibles de varier en fonction des systèmes de soins. Nous en voulons comme preuve le fait que le rapport de cote que nous avons obtenu avec ce facteur $(\mathrm{RC}=1,80)$, en suivant la résolution factorielle de Wright, va dans le sens contraire de celui observé dans l'étude originale ${ }^{[15]}$ $(\mathrm{RC}=0,37)$. Au Québec, plus du tiers $(39 \%)$ des généralistes restreignent leur pratique à une pratique hospitalière, comparativement à seulement $9 \%$ dans la province canadienne voisine d'Ontario ${ }^{\text {[27] }}$. Il est probable qu'une proportion importante d'étudiants québécois perçoit le choix d'une carrière en médecine familiale comme permettant l'accès à la pratique hospitalière qu'ils valorisent.

En ce qui concerne l'item «accent mis sur les soins d'urgence », qui contribuait davantage au facteur de «diversification du champ de pratique» qu'au facteur combiné « prestige et orientation hospitalière » dans nos analyses exploratoires, il est possible que les étudiants qui choisissent la médecine familiale perçoivent les soins d'urgence comme une possibilité de diversifier leur pratique en dehors du cadre de la pratique régulière en cabinet. La surreprésentation de ces étudiants dans notre échantillon pourrait donc expliquer pourquoi cet item présentait une association inattendue et marquée au facteur de «diversification du champ de pratique». Il faut toutefois rappeler que nos résultats ne sont pas en rupture complète avec ceux des auteurs du questionnaire original, puisque cet item dénotait également, dans nos analyses, une contribution importante au facteur auquel Wright et al. l'avaient associé. Par conséquent, nous recommandons le modèle à cinq facteurs proposé par les auteurs de la version originale du questionnaire pour les investigations futures, considérant que la validité prédictive de ce modèle était à toutes fins pratiques identique à celle du modèle à quatre facteurs obtenu dans nos analyses exploratoires.

Nous n'avons pas, à ce stade, réalisé d'analyses confirmatoires proprement dites (par équations structurales) sur les résolutions factorielles obtenues (à 4 ou 5 facteurs). D'une part, la taille de notre échantillon s'avérait limitée pour de telles analyses. D'autre part, notre échantillon était québécois et, nous l'avons vu, les distributions observées en matière de choix de carrière peuvent varier selon le contexte socioculturel, variable dans les pays francophones. À cet effet, ajoutons que certains pays tiennent un concours pour les spécialités au terme du premier cycle d'études médicales et que l'espoir de le réussir ou la crainte de l'échouer pourraient s'avérer être des items utiles à ajouter pour comprendre le choix de carrière des étudiants. Ces considérations 
seraient à prendre en compte lors d'adaptations et d'utilisations ultérieures du questionnaire.

\section{Conclusion}

En dépit d'un échantillon dont la composition différait substantiellement de celui de Wright et al., les facteurs qui se sont avérés influencer le plus significativement le choix d'une carrière en médecine générale chez les étudiants en médecine (l'orientation sociétale et la diversification du champ de pratique) ainsi que le pourcentage de la variance du choix de carrière expliquée $(\approx 50 \%)$ dans notre étude se sont avérés comparables aux résultats obtenus lors de l'étude originale effectuée avec le questionnaire anglais. Ceci plaide en faveur de la robustesse de l'instrument de mesure développé par Wright et al. et de la fidélité de la version française que nous avons développée. Bien que nous recommandions qu'une attention particulière soit portée à l'adéquation socioculturelle de la résolution factorielle proposée, notre traduction française du questionnaire de Wright peut être considérée comme étant aussi valide et fidèle que le questionnaire original et peut être utilisée dans le cadre d'études visant des répondants francophones, qu'il s'agisse d'enquêtes comparatives ou d'investigations pour suivre l'évolution des attitudes des étudiants durant le cursus médical, par exemple.

\section{Contributions}

Marie-Dominique Beaulieu et Jeannie Haggerty ont participé à la conception du protocole de recherche, au recueil de données, à l'analyse statistique et à l'interprétation des résultats et à la rédaction du manuscrit. François Goulet a participé à la conception du travail de recherche, à l'interprétation des résultats et à la rédaction du manuscrit. Fatima Bouharaoui a participé à l'analyse statistique et à l'interprétation des résultats.

La version française du questionnaire de Wright et al. est disponible auprès des auteurs, sur demande.

\section{Remerciements}

Nous remercions le docteur Bruce Wright pour nous avoir donné la permission d'utiliser et de traduire son questionnaire, ainsi que Monsieur Claudio Del Grande pour son appui à la préparation de ce manuscrit.

Cette étude a bénéficié d'une bourse JANUS 2004 du Collège des médecins de famille du Canada et a reçu l'approbation du Comité d'éthique sur la recherche chez les êtres humains de la Faculté de médecine de l'Université de Montréal.

\section{Références}

1. Rosser WW. The decline of family medicine as a career choice. CMAJ 2002;166:1419-20.

2. Fincher RM. The road less traveled-attracting students to primary care. N Engl J Med 2004;351:630-2.

3. Bowler I, Jackson N. Experiences and career intentions of general practice registrars in Thames deaneries: postal survey. BMJ 2002;324:464-5.

4. Soulier E, Grenier C, Lewkowicz M. La crise du médecin généraliste : une approche cognitive de la profession. Revue Médicale de l'Assurance Maladie 2006;37:99108.

5. Roupret M, Hupertan V, Chartier-Kastler E. The choice of a medical career in a population of 600 second-cycle French medical students preparing the national-ranking exam. Presse Med 2005;34:786-90.

6. Bland CJ, Meurer LN, Maldonado G. Determinants of primary care specialty choice: A non-statistical metaanalysis of the literature. Acad Med 1995;70:620-41.

7. Campos-Outcalt D, Senf J, Kutob R. A comparison of primary care graduates from schools with increasing production of family physicians to those from schools with decreasing production. Fam Med 2004;36:260-4.

8. Campos-Outcalt D, Senf J, Watkins AJ, Bastacky S. The effects of medical school curricula, faculty role models, and biomedical research support on choice of generalist physician careers: a review and quality assessment of the literature. Acad Med 1995;70:611-9.

9. Reed VA, Jernstedt GC. Understanding and improving medical student specialty choice: a synthesis of the literature using decision theory as a referent. Teach Learn Med 2001;13:117-29.

10. Senf JH, Campos-Outcalt D, Kutob R. Factors related to the choice of family medecine: a reassessment and literature review. J Am Board Fam Pract 2003;16:502-12. 
11. Beaudoin C, Maheux B, Béland F. Attitudes of students entering internships and residencies in family medicine. Can Fam Phys 1991;37:857-60.

12. Ward AM, Kamien M, Lopez DG. Medical career choice and practice location: early factors predicting course completion, career choice and practice location. Med Educ 2004;38:239-48.

13. Parlow J, Rothman AI. ATSIM: a scale to measure attitudes toward psychosocial factors in health care. J Med Educ 1974;49:385-7.

14. Murdoch MM, Kressin N, Fortier L, Giuffre PA, Oswald L. Evaluating the psychometric properties of a scale to measure medical students' career-related values. Acad Med 2001;76:157-65.

15. Wright B, Scott I, Woloschuk W, Brenneis F. Career choice of new medical students at three Canadian universities: family medicine versus specialty medicine. CMAJ 2004;170:1920-4.

16. College of Family Physicians of Canada, Canadian Medical Association, Royal College of Physicians and Surgeons of Canada. 2007 National Physician Survey: Medical Student Questionnaire, 2006 [On-line]. Disponible sur : http://www. nationalphysiciansurvey.ca/nps/2007-Q/ Student_Questionnaire_2007_e.pdf

17. Harvey A, DesCoteaux JG, Banner S. Trends in disciplines selected by applicants in the Canadian resident matches, 1994-2004. CMAJ 2005;172:737.

18. Choucair J, Nemr E, Sleilaty G, Abboud M. Choix de la spécialité en médecine : Quels facteurs influencent la décision des étudiants ? Pédagogie médicale 2007;8:14555 .
19. Observatoire National de la Démographie des Professions de Santé. Rapport annuel 2006-2007, Tome 1 : La médecine générale. Paris : ONDPS, 2008.

20. Vanderschelden M. Les affectations des étudiants en médecine à l'issue des épreuves classantes nationales en 2007. Études et résultats 2007; DREES(616).

21. Vercruysse B. Continuité des soins et garde de médecine générale en Belgique. Actes du colloque «Les médecins généralistes face à l'organisation de l'offre de soins ». Rennes : ENSP, 2006 :33-45.

22. Nabli AT, Bougmiza MI, Mtiraoui A. Attitudes of Tunisian medical students to medical practice: gender differences. East Mediterr Health J 2008;14:686-96.

23. Beaton DE, Bombardier C, Guillemin F, Ferraz MB. Guidelines for the process of cross-cultural adaptation of self-report measures. Spine 2000; 25:3186-91.

24. Daniel WW. Biostatistics: a foundation for analysis in the health sciences. 4th ed. New York: John Wiley and sons; 1987.

25. Jenks S. ACS keeps mammography guidelines for women under 50. J Natl Cancer Inst 1993;85:348-9.

26. Gosling SD, Vazire S, Srivastava S, John OP. Should we trust web-based studies? A comparative analysis of six preconceptions about internet questionnaires. Am Psychol 2004;59:93-104.

27. Fédération des médecins omnipraticiens du Québec. Énoncé de principes pour une politique nationale sur la médecine familiale. Montréal : FMOQ, 2008.

Correspondance et offprints : Marie-Dominique Beaulieu, Centre de recherche du Centre hospitalier de l'Université de Montréal, Hôpital Notre-Dame, Pavillon L.-C. Simard, $8^{\text {e }}$ étage, 1560 rue Sherbrooke Est, Montréal, Québec, Canada H2L 4M1 Mailto : marie-dominique.beaulieu@umontreal.ca 